\title{
Application of Surveying and Mapping and Digital Technology
}

\author{
Shumin Xu \\ Zhejiang Geological Institute of CMGB, Hangzhou, Zhejiang, 315012, China
}

\begin{abstract}
With the continuous development of the entire economy, society, and science and technology, the entire earth surveying and mapping technology is also constantly upgrading and developing. With the deepening of digitization, the development of digital surveying and mapping technology has gradually strengthened. Geographical surveying and mapping work plays an important role in the socio-economic development of the entire country and region. The essence and connotation of digital surveying and mapping technology is to serve geographic space and digital technology, and gradually develop to all aspects and deeper disciplines. In the surveying and mapping work, it is necessary to gradually improve the management of the entire digital surveying and mapping technology, continuously improve the service capabilities, and develop the entire surveying and mapping technology to a higher level.
\end{abstract}

\section{Keywords}

surveying and mapping; digital technology; application

\section{测绘学和数字化技术的应用}

\section{徐书敏}

中国冶金地质总局浙江地质勘查院，中国・浙江 杭州 315012

\section{摘 要}

随着整个经济、社会和科学技术的不断发展，整个地球测绘技术也在不断升级和发展。随着数字化的深入，数字化测绘技术 的发展也逐渐加强。地理测绘工作在整个国家和地区的社会经济发展中发挥着重要的作用。数字化测绘技术的本质和内涵是 服务于地理空间和数字技术。并逐渐向各个方面和更深入的学科发展。在测绘工作中, 要逐步完善对整个数字测绘技术的管理, 不断提高服务能力，把整个测绘技术发展到更高层次。

\author{
关键词 \\ 测绘学; 数字化技术; 应用
}

\section{1 工程测量中测绘学与数字化技术的应用}

\section{1 工程测量中测绘学与数字化技术分析}

由于数字化测绘技术的快速发展, 被广泛运用在工程建 设中, 对测量工作的开展有关键作用, 如 GPS、远程控制技 术等。在原来的测量工作时, 大部分是由人工完成的, 通过 自身的计算和测量完成这项工作，这样工作的弊端是时间耗 费较多, 无法满足我们的要求。数字化测绘技术的出现改变 这种局面, 通过运用这项技术对数据进行分析处理, 自动进 行绘图, 和传统的测绘比较, 数字化测绘技术明显有很大的 优点。数字化技术的飞速发展在工程建设中发挥重要作用, 但在某些方面还存在一些缺点, 所以应随时发现其不足及时 进行技术更新，促使这项技术更加符合实际需求。

\section{2 数字化测绘技术的优点分析}

与传统测绘技术相比数字化测绘技术优势非常多, 在工
程测量工作中的重要性比较明显。数字化测绘技术在工程测 量中应用以后，大大提高了测图精度，同时数据处理化程度 也提高了很多，这对于工程测量数据的有效管理至关重要。

\subsection{1 计算机模拟方面}

数字化测绘技术在具体应用过程中，可以利用计算机将 测量到的地形、地貌特征等相关信息更直观的呈现给相关工 作人员。传统测绘技术在应用过程中通常都会涉及大量的线 条、数字等元素, 而要想向判断出图中各元素的准确含义, 就要求其工作人员必须要拥有较高的专业素养, 而数字化测 绘技术在实际应用中，对工作人员的专业素养的要求则相对 较弱, 进而有效弥补了传统技术存在的不足。

\subsection{2 储存更加方便}

数字化测绘技术应用于工程测量中以后, 数字化产品的 保管、存放都非常方便，同时数字化产品信息的不变形性也 
得到了保存, 工程施工过程中地形图的实用性大大增强, 可 以有效避免重复测量的出现，大大降低了工程测量的成本， 经济效益也得到了提升。此外, 数字化测绘技术在工程测量 中应用以后, 地形信息存储更加方便, 可以随时对数据进行 处理, 地形信息的用途得到了扩展, 充分满足了客户的实际 需求。

1.2.3 能够满足用户的不同需求, 有助于提高自动化 程度

对于数字化测绘产品来讲, 不同用户对其都有不同需求, 数字化测绘技术能够结合客户需求的变化, 对相应产品实行 再加工, 进而充分满足客户对产品中各元素的要求。同时, 数字化测绘产品还具有拼接、缩放等功能, 而通过科学合理 的拼接能够不断拓宽其产品的应用范围。另外, 采用数字化 测绘技术成图后, 可以利用计算机在此图基础上, 实现不同 设计与规划。同时, 还可以将其与其他方案进行对比分析, 从而选择最佳实施方案。而在利用数字化测绘技术进行生产 时, 往往都需要利用计算机来实现, 在这种前提下, 其生产 过程中的自动化、准确性和规范性也会不断提升。

\subsection{4 图形信息的丰富化}

与传统的测绘技术比较, 数字化测绘技术在地图上测绘 的坐标更加准确、具体化, 描绘更加清晰, 可以将地形点上 的所有属性信息全面地展现出现, 同时在绘图的过程中更容 易对测图符号进行调用, 使地图地形的绘制更加丰富、全面、 规范。为工程测量以及绘图提供了便利。

\section{3 数字化测绘技术在工程测量中的应用}

\subsection{1 数字地面测图的应用}

数字化测图无论是优势还是发展前景都是传统的模拟测 图无法比拟的。在测量工程中, 地面数字化测图技术得到了 广泛的应用, 主要是因为其测量的精度比较高。同时由于地 面数字化测图技术可以很好地进行空间数据的采集存储、图 形绘制、成图输出, 并且还能够自动的生产精度比较高的数 字图。在搜集野外数据时, 数字测图能利用的设施有全站仪 和 GPSPTK 两种, 这两种设施进行数据搜集的方式不同, 且 每一种方式都有优势和劣势。和过去相比, 现代人们对于工 程质量的要求也在持续加强, 所以如果只是应用两种设施中 的一种, 已不能满足要求, 因此大多时候都是同时应用这两 种测量设施, 通常来说会应用 GPSPTK 实施测量, 而测量得
到的数据可以在全站仪中应用起来。通过全站仪测量得到的 数值也通常用来检验 GPS 测量结果的正确性, 由于同时应用 的缘故，全站仪所具备的水平方向遮挡问题包括 GPS 所具备 的上方遮挡问题都可以有效解决, 从而实现真正的优势互补。

\subsection{2 数字化成图技术应用}

数字摄影技术主要是通过利用计算机技术随影像进行数 字化处理和匹配, 并且它与视觉和信息的联系是非常密切的, 首先可以利用影像对三维表面模型进行创建, 然后根据模型 的实际情况进行图形测量和绘制。现在的数字化成图技术可 分为内外业一体化模式和电子平板模式。内外一体化模式就 是将内外业的有关技术结合在一起，共同应用到地图的绘制 当中, 在测量过程中应用全站仪及电子手簿来进行野外的数 据采集。这种测图技术能更大地提高测量准确度, 更好地提 高工作效率。电子平板测图技术就是在地图的绘制中, 结合 地图现有的相关信息资料, 根据有关要求规定, 系统自动地 对相关信息进行综合，从而呈现一种新型的电子地图绘制的 形式。但这种方式因为外部环境及实际地形考察数据误差, 会影响平板电子绘图的可靠性。

\subsection{3 数字化成像技术在工程测量中的应用}

数字化成像技术大多数都是应用在大比例尺的地图测量 工作当中, 应用空间数据的采集储存、图形测绘、成像输出 等一体化的测绘方式完成测绘工作。这一种方式的应用准确 度相对较高, 在工程测量当中的应用非常广泛。数字化测绘 技术可以应用一次性的测量获得最终的测绘结果, 并通过不 同比例尺的地图绘制，满足不同的专业人员对地图的应用需 求, 规避开展重复性的测量工作。在数据采集的过程中, 数 字化测绘技术还可以实现三维坐标的自动采集、储存和处理, 消除因为人为参与而导致的误差。对此, 数字化成像技术的 合理应用, 不仅可以提升测绘去准确度, 还可以节省人力物 力的投入。一般情况下, 只要是没有强烈磁场的地区, 基本 上数字化成像技术的应用可以说是任何障碍。

\subsection{4 数字化测绘技术在工程测量体现的价值}

数字化测绘技术在工程测量中的体现的价值主要体现在 以下方面: (1)数字化测绘技术中的遥感技术应用。遥感技术 是指通过遥感仪器等对测绘目标进行传感和探测, 获取探测 目标的真实情况，对目标的反射、散射等电磁波信息进行接 收、提取、转化、分析、处理和应用的一门技术。由于测绘 
的地形的面积较大, 需要进行同时探测, 数字化的测绘技术 因其综合性能强和适用范围广, 已为工程测量起到了重要作 用; (2)数字化测绘技术通过全球定位系统对测绘目标做定位 操作, 对采集数据进行整理、分析, 提取测绘数据; (3)数字 化的成图技术。测绘技术的成图应用, 可以将数据绘图, 图、 表、文字结合使得测绘技术得更加广泛应用与工程测量; (4) 数字摄影测量技术的应用。数字测绘技术将摄影与数字结合, 运用计算机网络技术进行处理和成图, 运用高科技技术和方 法进行协调和制作。将数字绘图应用得更加逼真和实用。数 字化测绘技术的不断提高和更新, 为工程测量这项工作提供 了便利和有效的价值, 也为施工工作提供便捷条件。

在工程测量中应用数字化测绘技术, 有利于提高测绘的 精度, 提供更加准确的地图信息, 结合现代科技, 降低工作 人员的时间和工作量, 提高工程测量的工作效率, 降低工程 测量的成本。数字测绘技术的应用越来越广泛, 数字化测绘 技术会不断发展，更好地为社会发展服务。

\section{2 数字化地籍测绘技术的运用}

\section{1 数字化地籍测绘技术的优势}

采用数字化的地籍测绘技术，可以实现对于数据信息的 数字化和自动化的管理。采用数字化的测绘技术, 主要是通 过对于数据的采集, 编码, 然后传输, 最后在进行存储, 通 过这样的过程来对数据以及图像进行处理, 然后最后再在电 脑上显示, 并且打印出来。数字化的地籍测绘产品还有更高 的技术含量, 而且更加精确, 而且用数字化的测绘方式可以 更好地减少误差, 可以更好地节省人力物力, 可以帮助人们 更好地对于数据进行管理。数字化的地籍测量主要是用计算 机来自动的进行记录, 处理, 自动生成图片, 总而言之, 具 有很强的自动化特点。而传统的地籍测量则主要采用经纬仪 与平板仪量角器相结合的方法进行操作。通过这样的方式很 难做到高精确度, 并不适合现代技术的发展。采用数字化的 地籍测量方式还可以对数据信息及时地进行更新, 使使用者 更好地了解最新的数据情况。通过计算机来展开数字化的地 籍测量, 把提高了地籍测量的效率, 而且使测量的数据更加 科学化, 更具有参考价值。

\section{2 数字化地籍测绘的作业方法}

\section{2 .1 控制测量}

对于地籍测绘来说, 控制测量也很重要。控制测量的主
要目的是为了让地物点和界址点更加准确, 能够让测图的精 度控制在一定的范围, 能够保障测量数据准确度和精准度。 由于 GPS 技术的发展, 所以减轻了测量工作的强度, GPS 控 制和灵活简便的导线替代传统的三角测量方法，可以使控制 工作变得更加的高效简洁, 主要是因为这 GPS 技术的普及才 能使地籍测量工作变得更加的方便, 高效。控制测量主要分 为首级控制测量, 图根控制测量。首级控制测量指的是通过 静态 GPS 技术鉴定, 然后用随机评查软件在进行严密平差。 图根控制测量主要采用的是导线测量和各种交会测量相结合 的方式。这两种控制测量的方法可以使测量数据更加准确, 更符合要求。

\section{2 .2 细部点的测量}

(1) 扫描数字化作业

所谓扫描数字化作业指的是将图纸上的测图, 通过扫描 的方式, 然后得出数字化的测量图, 而其实真正操作起来并 没有这么简单, 还是有很高的要求。必须对已有的地形图或 者地籍图首先进行扫描, 然后得到一个栅格图形, 然后再利 用专业的扫描矢量化软件进行扫描, 从而将栅格图形转变成 一种矢量图形, 这样才能将原有的地籍图更加数字化。但是 通过扫描原有的地基图来得到数字化的数据, 这样的方式并 不具有很高的准确率, 可能会出现一些数字上的偏差, 例如 在一些地物点和界址点的坐标上, 可能会出现误差, 因此需 要再用修测和补测的方法来对其进行修正, 通过这样的方式, 才能更好地提高数字化地籍图的精确度。

\section{(2) 全数字摄影测量技术}

第二种就是通过摄像的方式来获取数字化数据, 主要是 通过专门的航测软件来对物象进行拍摄, 然后再形成与之匹 配的数字模型, 最后再通过专业的软件来获得数字地图, 通 过这样的方式可以更好地弥补扫描式的数字化地籍测量的缺 点。通过这样的方式, 可以将大量的在外进行的工作转移到 室内, 可以更加方便地在室内完成工作。而且通过这样的测 量方式, 可以更好地减轻工作人员的压力, 可以以速度最快, 然后精确度最高, 而且成本最低的方式来获取所需要的数据。 全数字摄影测量技术必定会成为今后数字测量的一个重要方 向, 因此还需要更加去完善这种测量方法。

总之, 数字化的地籍测量不仅是要形成一幅图片, 更重 要的是形成一个数据库, 这个数据库包含许多内容, 主要包 
括一些宗地数据信息, 还有一些宗地的面积和街道的分布, 以及界址点坐标和地物点的坐标等等, 通过这个数据库, 可 以更好地让政府以及相关人员对于中国的土地情况有一个基 本的了解。通过数字化的地籍测量方式, 可以为中国的土地 管理方面提供很大的帮助, 因此需要不断地发展和完善。

\section{3 在建筑工程领域的运用}

\section{1 建筑工程领域的测绘学与数字化技术分析}

\section{1 .1 信息化技术}

数字化测绘当中由于加入了先进的信息化技术, 自动化 操作程度高, 从而之在建筑工程测量上操作水平高, 测绘产 品当中都含有相当的信息化功能，还可以根据测绘的情况加 以修改和补充, 产生出新的图来供给使用。另外还可以对测 量的数据进行自动的修正, 提高了测绘产品的质量。数字化 测绘技术相关的产品在使用的各阶段都具有信息化的特点。

\section{1 .2 图形处理技术}

数字化技术的测绘产品全部都拥有图形处理技术，可以 按不同的需求进行相关的图形处理, 包括对图形进行拼接、 缩放甚至对产品的各种要素进行数据再加工。图形处理技术 大大提高了地形图的作用, 还简化了工作流程, 从而可以增 加大量的用户，最终起到了提高企业效益的目的。

\subsection{3 数字化技术}

数字化技术相比传统的测绘技术, 在测绘的精度上可以 说是起到了关键作用。测绘精度得到了质地提高, 大大提高 了测绘结果的准确性和实用性。由于是采用的数字化技术, 减少了人工的操作过程, 减少了工作误差, 保证了测量结果 的精准度。另外一方面在实施测绘的过程当中, 数字化技术 还可以减小工作人员的工作量, 提高了工作效率, 降低了生 产成本。

\section{2 技术应用及其发展}

\subsection{1 数字化成图技术}

在以往的工程测绘工作，大比例尺图形的测绘以及工程 图的测绘有着非常重要的地位, 但是这些测绘工作需要进行 大规模的野外作业, 这就加大了测绘人员的工作量与工作强 度, 增加了工程测绘的成本, 但应用数字化成图技术就可以 解决这一问题。测绘技术经过不断地改进与优化, 越来越趋 于完善、成熟, 与传统的测绘工作相比, 数字化成图不需要
测绘人员进行大规模野外作业, 这就在很大程度上提高了出 图效率、减轻了测绘人员的工作强度，同时，也有利于改善 作业环境。作为一种新兴的测绘技术, 数字化成图具有劳动 量小、测绘便捷、准确度相对较高以及精度高的显著优势, 近年来在工程测量领域之中得到了越来越广泛的应用。就目 前的情况来看, 数字化成图可以分为 2 种模式, 一种是内外 一体化成图，另一种是电子平板成图。其中，内外一体化成 图的应用范围较为广泛, 其是一种较为常用的数据收集手段, 具有分工明确、精度高的显著优势, 且成图效率也比较高。

\subsubsection{RS 测绘技术的应用}

RS 测绘技术指的是 “遥感技术” ，该项技术主要是将 航空摄影技术作为基础，然后利用卫星实时观测地理信息。 在具体的测绘工作中, 利用 RS 技术能够实现在大面积范围 内同步观测地理信息, 这就在很大程度上保证了数据的有效 性和综合性，对于测量具有重要的意义。现今，RS 技术已经 在建筑工程测量中得到了广泛的应用，不仅能够大面积范围 内同步观测数据，而且还能够有效收集中小比例尺的图形数 据，这就为城市基础图形的测量工作奠定了良好的基础。RS 是现今最合适的观测基础地理信息的方法, 很多新型的测量 技术也是依照 RS 技术来研发的，由此可见， RS 技术在建筑 工程测量中具有举足轻重的作用。

\subsection{3 全球卫星定位技术的应用}

测绘新技术在建筑工程测量中的应用思路研究, 其次还 体现在全球卫星定位技术的应用方面。全球卫星定位技术的 应用，不仅在工程测量方面得到了应用，而且还在城市规划 以及工程测量方面得到了广泛的应用。全球卫星定位技术是 一种集计算机科学、空间科学信息科学、测绘遥感科学、环 境科学和管理科学等学科为一体的新兴学科。在众多领域的 应用和发展，足以凸显出其重要性。全球卫星定位系统在建 筑工程中的应用，使得中国的测绘定位技术发生了革命性的 变化与发展, 也使得中国的工程测量服务领域扩展到了经济 发展的各个部门。全球定位系统的应用, 使得工程项目的各 种信息得到全面的覆盖，提高了工程施工的效率，降低了工 程施工的工期。全球定位系统在工程项目中的应用，可以防 止数据的丢失, 防止数据被盜取, 提高数据的有效性。因此说, 全球定位系统在工程测量中的应用, 能有效地提高工程测量 的有效性，能够实现管理的科学化、标准化、信息化。 


\subsection{4 摄影测绘技术的应用}

摄影测绘技术指的是通过摄影来采集所需的数据信息的 一种技术。随着现代信息的不断发展，摄影测绘技术已经被 广泛运用到各个领域，逐步发展为数字化的摄影测绘技术。 在实际的建筑测量工程中, 数字化摄影测绘技术往往能够在 建筑密集的地区发挥重要的作用, 高效的大面积绘图能够为 测绘人员提供全面的城市建筑信息, 这就大大提高了建筑工 程测量的工作效率。除此之外, 摄影测绘技术还具有节约成 本的优点, 在建筑施工中尽可能地节约测量成本和对劳动力 的使用, 为建筑施工方节省更多的资金, 达到更好的经济效益。

\section{3 .5 发展方向}

在技术水平不断发展的今天, 工程测量数据是用之前的 测量场景的交互式场景的测量形式，收集到远程控制类型。 同时, 该平台还将根据施工现场的特殊要求测量工作, 从固 定地面变为机载、卫星控制, 逐步由静态变为动态, 极大地 提高了测量的灵活性。针对一些大型的比较复杂的建筑结构、 建筑工程的三维测量、现代工业生产自动化、工艺控制、产 品质量检测和监测数据及定位要求。精度要求越来越高, 很 好地实现了对三维测量技术向人类科学测量的推动延伸。其 主要的优点可以更好地促进中国建筑行业的发展, 故而在现 阶段得到了广泛的应用。

\section{4 测绘技术与数字化在油田勘测领域的应用}

\section{1 数字化测绘技术应用步骤}

油田勘探项目的要求不同, 测绘的目标和方案就不同, 当然所使用的仪器也不同。一般而言，在勘探项目的前期阶 段, 可以通过航空摄影技术 (RS) 对工区进行测绘工作, 结 合测绘获得的各种数据信息, 对工区的情况有一个初步了解, 再设计物理点的点位, 然后根据勘探项目的工作量、精度等 各种要求, 最后设计详细的测绘方案。

\section{2 测量方式}

在野外进行测绘时，首先可以利用全球卫星定位技术 (GPS) 建立 GPS 控制网, 将野外获得的一些静态 GPS 数据 进行分析和处理, 对国家控制点进行加密操作, 将这些控制 点当作测区的基本控制，或使用多基站网络 RTK 技术构建一 些基准站, 将已获得的物理点坐标上传到测绘仪器上, 相关 人员就可以进行标注、绘制等工作。

\section{3 数据处理分析}

数字化测绘的各种数据可以上传到计算机中, 然后相关 人员可以使用一些专业软件对这些数据进行相应的整理、分 析以及处理。专业人员在对这些数据进行相关的操作之后, 可以评判其是否符合要求。因为这些数据都是数字化的成果, 因此处理起来十分简便、误差较小，效率也非常高。

\section{4 实际应用}

\subsection{1 航空摄影与遥感}

在油田勘探过程中, 首先应该对研究区的地形地貌等情 况进行初步了解，才可以进行方案设计和工程作业准备。以 前都是委派工作人员深入现场进行踏勘, 不仅费时费力, 而 且踏勘的面积较小, 不够深入和全面, 特别是一些偏远的地区。 通过航空摄影技术 (RS) 对研究区进行航测, 不仅可以为工 程项目提供详细的地形地貌等情况，而且还能了解其他各种 信息，如交通、水域、植被等，为后续的设计、作业等提供 了极大的便利。

\subsection{2 全球定位系统}

目前，全球卫星定位技术（GPS）已在各行各业中得到 了十分广泛的应用，在油田勘探中也不例外，尤其是其中的 静态后处理技术，该技术主要作用是构建研究区的控制网以 及对测线端点进行静态检核，在一定程度上大幅提高了测绘 作业的精度。通过静态后处理技术，测绘小组流动可实时接 收信号, 并进行分析和计算, 获得准确的坐标和高程信息, 并且根据采集各种数据, 利用相关专业软件能够绘制各种图 件，极大地提高了测绘的效率和质量。

\subsection{3 地理信息系统}

除了上述两种技术之外，地理信息系统技术（GIS）也 逐渐在油田勘探中得到了应用，其应用主要分为以下三个方 面：(1)绘制各种图件，如作业图、地形地貌图、交通图以及 障碍物图等, 能够为油田勘探作业提供比较科学、实用的参考; (2)三维显示一些图件，能够立体、多角度地展示研究区的各 种情况; (3)处理、储存一些测绘数据, 并且使其得到有效的 应用。

实际上，地理信息技术的效用为让相关工作人员掌握测 绘地区的地理信息，从而制定行之有效的决策。此种地理信 息技术还被叫作环境信息系统与资源信息系统，这是因为此 种技术经常被应用到地球环境与资源现状的反映上，合理应 
用现代化技术，把计算机系统同数据库系统有机结合起来， 让所获的信息更加全面、完整，有助于工作人员掌握与应用， 可以统一遥感技术远距离通信设备的性能, 通过科学的手段 将勘测和定位问题优化，展开实时测绘工作，增强信息资源 的真实性, 为测绘工作争取更大的空间。

可以说，当前应用最广泛的技术便是 GPS 技术，其实就 是全球定位技术，此测绘技术是从定位技术延伸而来的，起 初应用在军事上。在油田测绘中, 合理应用 GPS 技术可以实 现动态监测油田地质, 因为不断地测绘, 让测绘技术的实效 性得到了明显提高, 为了油田建设提供了准确的参考数据。 另外, 在实际测绘中, GPS 技术可以将测绘项目完全覆盖住, 保证测绘工作全方位, 并能够把所测的数据第一时间传输到 客户端, 客户能够直接利用软件将对应的测绘结果计算出来, 切实提高测绘工作质量与水平。

\subsection{4 影像定位技术}

一些由岩石组成的地质, 若想对其进行测量, 需要合理 应用影像定位技术。通过图像化与数字化对地质信息进行有效
处理, 得出最终测量结果。另外, 还能应用一些其他新兴技术, 比如遥感影像技术来联合应用，此种技术能够有效定位影响， 将地质测量结果与周边环节直接呈现出来, 与传统技术相比, 信息呈现的要更加具体, 便于工作人员全面掌握矿山地质状况。

\section{参考文献}

[1] 刘群. 现阶段数字化测绘技术在工程测量中的应用 [J]. 城市地 理 ,2015(10): 18-20.

[2] 张玉丽. 数字化测绘技术在地籍测量中的应用 [J]. 中国新技术新 产品 ,2018,No.373(15):111-112.

[3] 殷海明. 数字化测绘技术在工程测量中的应用研究 [J]. 工程建设 与设计, 2018(6):263-264.

[4] 王春光. 从数字化测绘到信息化测绘的测绘学科新进展 [J]. 科技 视界, 2015(4):392

[5] 王恒. 数字化地形测绘常见问题分析 [J]. 华东科技: 学术 版, 2017(4):356.

[6] 王瑞丰. 数字化测绘技术在地籍测量工程中的应用思路 [J]. 统计 与管理 , 2014(5):153-154. 